\title{
Observation of Ultrafast Nonequilibrium Collective Dynamics in Warm Dense Hydrogen
}

\author{
R. R. Fäustlin, ${ }^{1}$ Th. Bornath, ${ }^{2}$ T. Döppner, ${ }^{3}$ S. Düsterer, ${ }^{1}$ E. Förster, ${ }^{4}$ C. Fortmann, ${ }^{5}$ S. H. Glenzer, ${ }^{3}$ S. Göde, ${ }^{2}$ G. Gregori, ${ }^{6}$ \\ R. Irsig, ${ }^{2}$ T. Laarmann, ${ }^{1}$ H. J. Lee, ${ }^{7}$ B. Li,${ }^{8}$ K.-H. Meiwes-Broer, ${ }^{2}$ J. Mithen, ${ }^{6}$ B. Nagler, ${ }^{6}$ A. Przystawik, ${ }^{2}$ H. Redlin, ${ }^{1}$ \\ R. Redmer, ${ }^{2}$ H. Reinholz, ${ }^{2}$ G. Röpke, ${ }^{2}$ F. Tavella, ${ }^{1}$ R. Thiele, ${ }^{2}$ J. Tiggesbäumker, ${ }^{2}$ S. Toleikis, ${ }^{1}$ I. Uschmann, ${ }^{4}$ S. M. Vinko, ${ }^{6}$ \\ T. Whitcher, ${ }^{6}$ U. Zastrau, ${ }^{4}$ B. Ziaja, ${ }^{9,10}$ and Th. Tschentscher ${ }^{11, *}$ \\ ${ }^{1}$ DESY, Notkestr. 85, 22607 Hamburg, Germany \\ ${ }^{2}$ Institut für Physik, Universität Rostock, 18051 Rostock, Germany \\ ${ }^{3}$ L-399, LLNL, University of California, P.O. Box 808, Livermore, California 94551, USA \\ ${ }^{4}$ IOQ, Friedrich-Schiller-Universität, Max-Wien Platz, 1, 07743 Jena, Germany \\ ${ }^{5}$ Department of Physics and Astronomy, University of California Los Angeles, Los Angeles, California 90095, USA \\ ${ }^{6}$ Clarendon Laboratory, University of Oxford, Parks Road, Oxford OX1 3PU, United Kingdom \\ ${ }^{7}$ LCLS, SLAC National Accelerator Laboratory, Menlo Park, California 94025, USA \\ ${ }^{8}$ Central Laser Facility, Rutherford Appleton Laboratory, Didcot, OX11 0QX, United Kingdom \\ ${ }^{9}$ CFEL, Notkestr. 85, 22607 Hamburg, Germany \\ ${ }^{10}$ Institute of Nuclear Physics, Radzikowskiego 152, 31-342 Krakow, Poland \\ ${ }^{11}$ European XFEL GmbH, Albert-Einstein-Ring 19, 22761 Hamburg, Germany
}

(Received 29 October 2009; published 25 March 2010)

\begin{abstract}
We investigate ultrafast (fs) electron dynamics in a liquid hydrogen sample, isochorically and volumetrically heated to a moderately coupled plasma state. Thomson scattering measurements using $91.8 \mathrm{eV}$ photons from the free-electron laser in Hamburg (FLASH at DESY) show that the hydrogen plasma has been driven to a nonthermal state with an electron temperature of $13 \mathrm{eV}$ and an ion temperature below $0.1 \mathrm{eV}$, while the free-electron density is $2.8 \times 10^{20} \mathrm{~cm}^{-3}$. For dense plasmas, our experimental data strongly support a nonequilibrium kinetics model that uses impact ionization cross sections based on classical free-electron collisions.
\end{abstract}

PACS numbers: 52.20.Fs, 52.25.Os, 52.27.Gr, 52.50.Jm

The investigation of warm dense matter (WDM) is one of the grand challenges of contemporary physics [1]. WDM is a plasma state characterized by moderate-tostrong interparticle coupling which takes place at freeelectron temperatures of several $\mathrm{eV}$ and free-electron densities around solid density [1]. It is present in many physical environments, such as planetary interiors [2,3], gravitationally collapsing protostellar disks, laser matter interaction and particularly during the implosion of an inertial confinement fusion capsule [4]. While in the astrophysical context WDM exists under stable conditions, in the laboratory it is achieved only as a transient state bridging condensed matter and hot plasma regimes. Here, we report on the first investigation of the nonequilibrium transition of hydrogen from a liquid to a moderately coupled plasma on the fs time scale, induced by highly intense soft-X-ray irradiation. This is an important step towards the investigation of strongly-coupled plasmas which are within reach of current light sources such as the Linac Coherent Light Source (LCLS). Our measurement enables unprecedented direct tests of nonequilibrium statistical models beyond mean field theories in a regime where collision and relaxation processes are dominant [5-7].

The use of x-ray scattering for the investigation of dense, strongly-coupled plasmas was successfully demonstrated in the past decade [5,7-11]. This technique is the $\mathrm{x}$-ray analog of optical Thomson scattering (TS) [12] and enables the experimental determination of plasma parameters in dense systems where optical light cannot penetrate. While previous experiments were carried out using highenergy laser facilities, the advent of soft- and hard-x-ray free-electron lasers (FELs) makes ultrashort high brightness beams available for this type of research $[13,14]$. This Letter reports on ultrafast heating of liquid hydrogen and TS measurement of dense plasma parameters using soft$\mathrm{x}$-ray FEL radiation. For the first time, nonequilibrium distributions are observed and the underlying relaxation dynamics are compared with kinetic models showing electron relaxation times in the order of $20 \mathrm{fs}$, thus, shorter than the pulse duration.

The scattering taking place is collective TS, which is characterized by a spectrally blue and red shifted response due to collective electron motion, plasmons, and nearly elastic scattering due to ion acoustic fluctuations $[7,15]$. The frequency shift $\omega$ of the plasmons with respect to incident radiation is described by the dispersion relation $\omega^{2} \approx \omega_{p}^{2}+3 T_{e} k^{2} / m_{e}$, their intensity ratio by the detailed balance relation $S_{\text {blue }} / S_{\text {red }}=e^{-\hbar \omega / T_{e}}$, where $\omega_{p}=$ $\sqrt{n_{e} e^{2} / m_{e} \varepsilon_{0}}$ is the plasma frequency, $k$ the photon wave vector change, $m_{e}, n_{e}, T_{e}$ electron rest mass, density and temperature (in $\mathrm{eV}$ ), $e$ the electron charge, and $\varepsilon_{0}$ the vacuum permittivity. Therefore, from the measured asym- 
metry and frequency position of the plasmons we can determine the electron temperature via detailed balance and density via the above dispersion relation.

Our experiments have been performed at DESY using the free-electron laser in Hamburg (FLASH) [16,17] at a photon energy of $91.8 \mathrm{eV}$. FEL radiation with $5 \mathrm{~Hz}$ pulse repetition rate, average pulse energy on target of $15 \mu \mathrm{J}$ and duration of $\sim 40 \mathrm{fs}$ is focused to a $25 \mu \mathrm{m}$ spot using an elliptical mirror, yielding intensities of $\sim 8 \times$ $10^{13} \mathrm{~W} \mathrm{~cm}^{-2}$. The energy of each pulse is measured using a residual gas ionization detector [18] and fluctuates less than $10 \%$. Figure 1 shows a schematic of the experiment. The FEL pulses hit the liquid hydrogen jet which has a diameter of $20 \mu \mathrm{m}$, an atomic density of $4.2 \times 10^{22} \mathrm{~cm}^{-3}$, and a temperature of $2 \mathrm{meV}$, prepared in a liquid helium cooled cryostat [19]. With $60 \mathrm{~ms}^{-1}$ flow velocity of the hydrogen jet each pulse scatters from an unperturbed region of the sample. During hydrogen injection the chamber was at a pressure of $\sim 10^{-5}$ mbar. Scattering from the hydrogen jet is collected at $90^{\circ}$ relative to the incident FEL radiation and in the vertical plane since the FEL radiation is horizontally polarized. We use a variable line space grating spectrograph [20] with a resolution of $\lambda / \Delta \lambda=180$. A toroidal mirror $25.5 \mathrm{~cm}$ from the jet provides a collection solid angle of $1.9 \times 10^{-3} \mathrm{sr}$. Reference measurements of the FEL spectrum (Fig. 1) were performed using a second spectrograph, introduced into the FEL beam during tuning and setup, or a third spectrograph [21] which simultaneously records radiation passing by the liquid jet target.

For these experiments we have chosen $91.8 \mathrm{eV}$ soft-xray radiation to be in a regime where the photon energy is well above the plasma frequency for liquid density hydrogen $\left(\hbar \omega_{p}=7.6 \mathrm{eV}\right)$ and to match the large penetration depth of $9.4 \mu \mathrm{m}$ [22] to the target radius $(10 \mu \mathrm{m})$. While at this photon energy attenuation via photoabsorption has the highest cross section, a small fraction of the incident radiation is Thomson scattered. The ratio between these

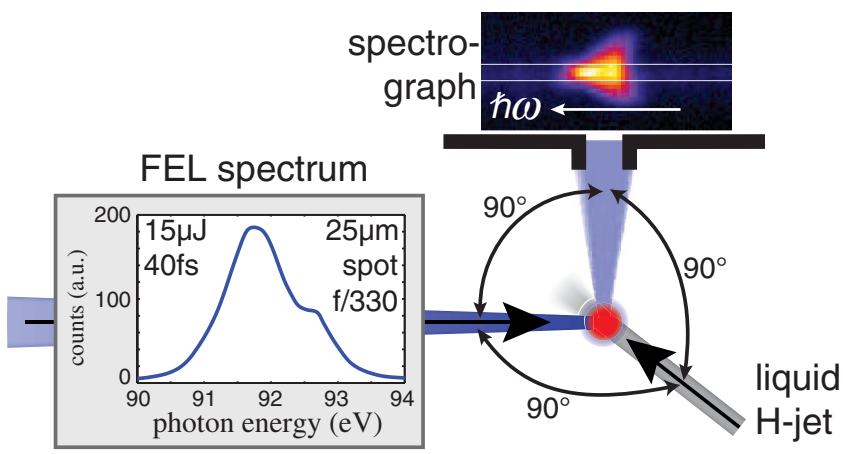

FIG. 1 (color online). Experimental setup showing the liquid hydrogen jet in the focus of the FEL beam with typical time averaged spectrum, and the spectrograph at $90^{\circ}$ scattering angle with the lineout region of the $\mathrm{CCD}$, corresponding to the focussed part of the source image, marked by white bars. two cross sections is $3.8 \times 10^{4}$ at $91.8 \mathrm{eV}$ [22]. Thus, the FEL pulses deposit energy in the liquid hydrogen while simultaneously probing the system. Because of the ultrashort pulse duration and the high penetration depth, the liquid hydrogen is heated isochorically and volumetrically by the pulse [23].

A scattering spectrum for 15 min integration time (4500 pulses) is shown in Fig. 2. It is composed of asymmetric peaks (plasmons) equally blue and red shifted from the incident photon energy by $0.65 \mathrm{eV}$. The measured spectrum is mainly broadened by the incident FEL bandwidth (1.1 eV full width at half maximum, mainly Gaussian, Fig. 1). Further source broadening effects $(0.2 \mathrm{eV}$ corresponding to the $20 \mu \mathrm{m}$ source diameter) are minimal due to the geometry of the setup and the FEL pointing stability.

The total spectrum is described by the dynamic structure factor $S(\omega)$ [24,25]:

$$
S(\omega)=Z_{f} S_{e e}(\omega)+Z^{2}\left(1-e^{-2 W}\right) S_{i i}(\omega) .
$$

Here, $Z$ is the nuclear charge and $Z_{f}$ the average number of free electrons per atom. The first term in Eq. (1) describes the high frequency free-electron fluctuations which includes collective plasmon scattering. The second term describes the ionic, nearly elastic response with the ion-ion structure factor $S_{i i}$ modulated by the Debye-Waller factor $1-e^{-2 W} \simeq 2 W \propto T_{i}^{2} / T_{D}^{2}$ [26,27], which accounts for low frequency ion acoustic fluctuations coupling with ionic thermal vibrations, where $T_{i}$ is the ion and $T_{D}$ the Debye temperature. In this experiment $T_{i}$ is sufficiently small against $T_{D}\left(T_{i} \sim 2 \mathrm{meV}\right.$, and $T_{D} \sim 10 \mathrm{meV}$ estimated using the Bohm-Staver relation [28]) so that elastic scattering is strongly suppressed. This effect was previously observed in laser based nonequilibrium experiments [29].

Collective TS spectra are calculated in Born-Mermin approximation [30] and convolved with the experimental

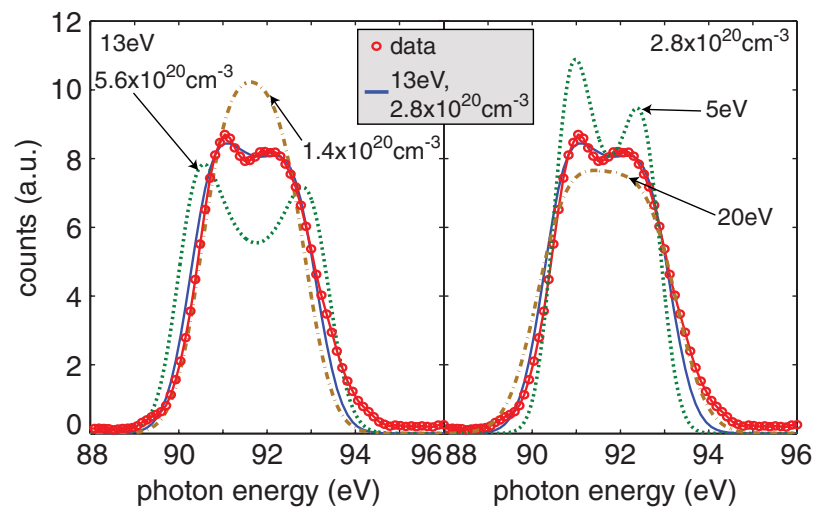

FIG. 2 (color online). Experimental spectrum (red circles) and the best fit of a calculated spectrum with $n_{e}=2.8 \times 10^{20} \mathrm{~cm}^{-3}$ and $T_{e}=13 \mathrm{eV}$ (solid blue line). Comparison to fits with variation in density (left graph), $5.6 \times 10^{20} \mathrm{~cm}^{-3}$ (dashed green line) and $1.4 \times 10^{20} \mathrm{~cm}^{-3}$ (dash-dotted brown line), and in temperature (right graph), $20 \mathrm{eV}$ (dash-dotted brown line) and $5 \mathrm{eV}$ (dashed green line), are shown. 
resolution. A synthesized spectrum fits the measurement best using an electron temperature and density of $13 \mathrm{eV}$ and $2.8 \times 10^{20} \mathrm{~cm}^{-3}$, respectively (Fig. 2). Spectra obtained by varying these parameters by a factor of 2 deviate considerably from the observed spectra. Sensitivity analysis shows that the error in the fitting procedure is $25 \%$ and $7 \%$ in electron temperature and density, respectively. Furthermore, elastic scattering is practically absent in the experimental spectra and comparing with synthesized spectra we obtain an upper limit for $T_{i}<0.1 \mathrm{eV}$, where $S_{i i}$ has been estimated using the screened one component plasma model [31]. Consequently, the liquid hydrogen is excited to a nonequilibrium plasma state with different electron and ion temperatures at a very low degree of ionization $(\sim 0.7 \%)$ but high atomic density $(4.2 \times$ $\left.10^{22} \mathrm{~cm}^{-3}\right)$.

The degree of ionization remains low despite the high electron temperature, due to the short time scale of the interaction. The electron-atom, electron-ion, and electronelectron energy transfer time scales for $13 \mathrm{eV}$ electrons are $>13 \mathrm{ps}, \sim 16 \mathrm{ps}$, and $\sim 25 \mathrm{fs}$, respectively [32]. Impact ionization takes $0.4-1 \mathrm{fs}$ [33,34], being the fastest process for electrons between $20 \mathrm{eV}$ and $400 \mathrm{eV}$. This suggests that the electronic subsystem is thermalized within the duration of the FEL pulse [Fig. 3(b)], but no equilibration has been reached between the other components explaining the combination of low degree of ionization and high electron temperature.

We have simulated the evolution of the electron kinetic energy distribution during and after the FEL irradiation (Fig. 3), using a model [35-37] based on kinetic equations. Because of the almost uniform target conditions (target radius matches penetration depth) [23] we can simulate a smaller target, a cluster of only $\sim 10^{5}$ hydrogen atoms. The simulation includes the following predominant interactions: photo- and collisional ionization, three-body recombination, elastic electron-ion and electron-atom scattering, and screened electron-electron interactions. Our model follows the full dynamics of an irradiated sample, from the nonequilibrium up to the thermal equilibrium phase for electrons. The electron temperature from the simulation $T_{e}^{\prime}=\frac{2}{3}\left\langle E_{\mathrm{kin}}^{e}\right\rangle$ is $12 \mathrm{eV}$, averaged over the FEL pulse duration, and the degree of ionization is $0.5 \%$, where $\left\langle E_{\mathrm{kin}}^{e}\right\rangle$ is the instantaneous ensemble average of the free-electron kinetic energy. These results compare well to the TS measurement. Furthermore, the electron energy distribution approaches a Maxwell-Boltzmann (MB) fit $\sim 20$ fs before the peak of the FEL pulse [Fig. 3(b)]. This assures that detailed balance is indeed applicable to evaluate the experimental spectrum.

Simulating the target interaction we can study the influence of different cross sections for impact ionization in dense plasmas by comparing with our measurement. We used the National Institute of Standards and Technology (NIST) database for molecular hydrogen [34] as well as an
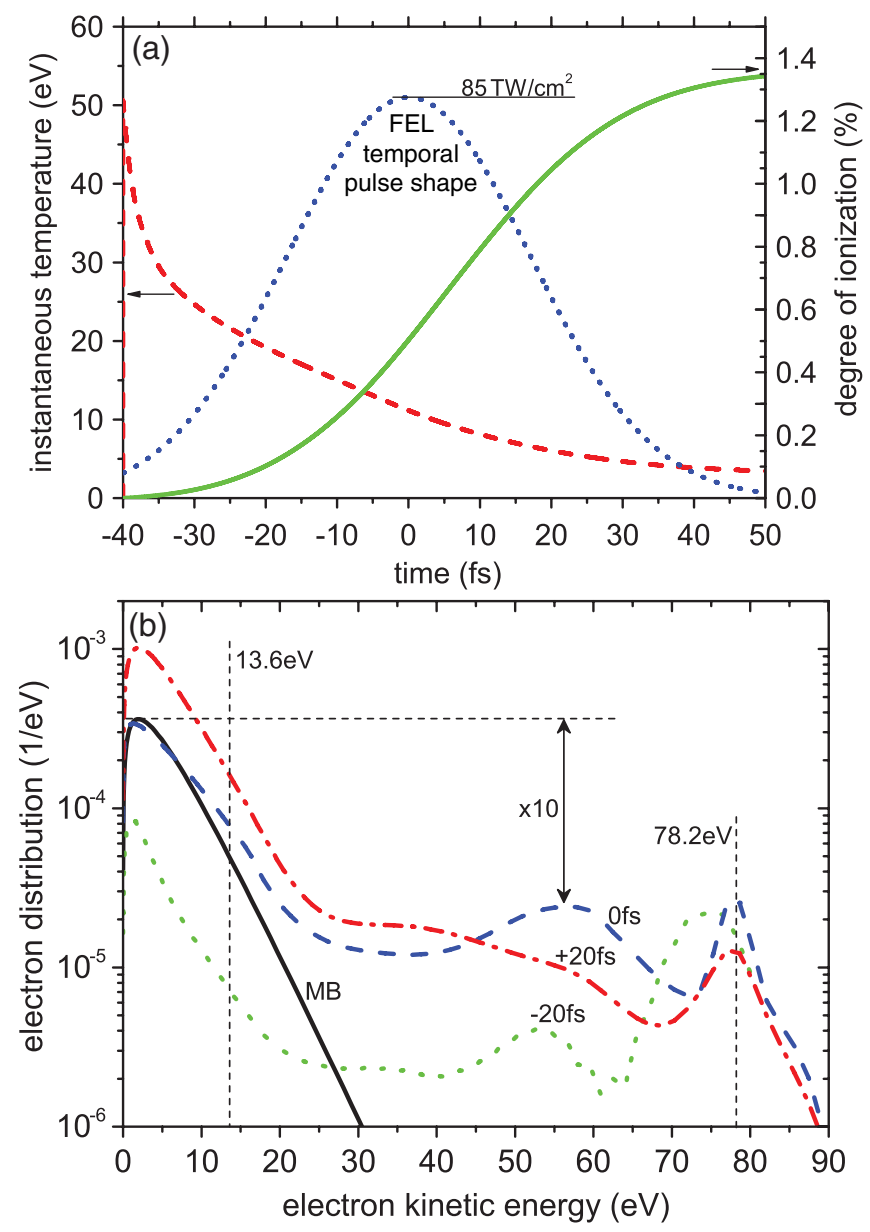

FIG. 3 (color online). Cluster simulation (a) with the FEL temporal profile (dotted blue line) and the evolution of the ionization degree (solid green line) as well as the electron temperature $T_{e}^{\prime}$ (dashed red line) using the Lieberman and Lichtenberg impact ionization. The electron kinetic energy distribution (b) $20 \mathrm{fs}$ before (dotted green line), at (dashed blue line), and 20 fs after (dash-dotted red line) the FEL pulse peak. A Maxwell-Boltzmann distribution fit at 0 fs (solid black line) and the photoelectron $(78.2 \mathrm{eV})$ and ionization $(13.6 \mathrm{eV})$ energies are shown for comparison.

expression from Lieberman and Lichtenberg [33]. The latter approximates the ionization via the low angle scattering of two free electrons and was employed in Fig. 3. NIST uses the binary encounter Bethe (BEB) model taking atomic and molecular structure into account. The models deviate up to a factor of 4 in the relevant electron energy range. Simulations with the classical model [33] yield an electron density and temperature at the FEL peak $(0 \mathrm{fs})$ which matches our measurement significantly better than BEB (Fig. 4). Even assuming a 4 times higher FEL incident flux cannot explain the observed values. A possible interpretation is that the atomic structure (as treated in BEB) does not play a significant role in the context of dense plasmas where, due to screening and correlation effects, 


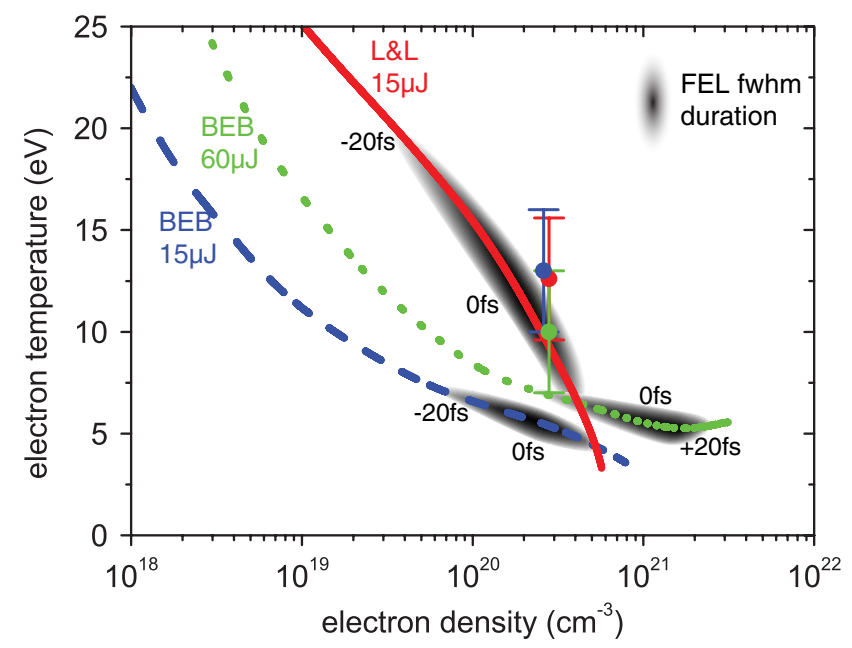

FIG. 4 (color online). Measurements (colored circles) compared to the simulated evolution of the target's free-electron density and temperature using different impact ionization cross sections and FEL pulse energies: Lieberman and Lichtenberg (solid red line, $15 \mu \mathrm{J}$ ) and the BEB model $(15 \mu \mathrm{J}$ dashed blue line, $60 \mu \mathrm{J}$ dotted green line). The FEL full width half maximum duration is indicated in gray scale.

high lying atomic states are removed and the electron interaction is more properly described with a classical ionic background.

In conclusion, we were able to obtain Thomson scattering spectra with an unprecedented signal to noise ratio due to the high repetition rate of the FEL and compared to single laser pulse driven $\mathrm{x}$-ray sources [8-10]. The ultrashort high intensity soft-x-ray pulses allow us to heat liquid hydrogen isochorically and volumetrically to the observed moderately coupled nonequilibrium state. Our results compare well to nonequilibrium kinetic simulations using classical impact ionization models which show an electron relaxation time scale of $\sim 20$ fs. Already available pumpprobe techniques [38] will allow the measurement of time scales for the subsequent relaxation channels via electronion equilibration. This is a pioneering step towards the investigation of nonequilibrium and strongly-coupled plasmas with implications from astro- to energy physics [1].

We thankfully acknowledge financial support by the Helmholtz Gemeinschaft via the Virtual Institute VH-VI104, the German Federal Ministry for Education and Research via Project No. FSP 301-FLASH, and the Deutsche Forschungsgemeinschaft (DFG) via the Sonderforschungsbereich SFB 652. T. L. acknowledges DFG support under Grant No. LA 1431/2-1, R. R. F. under Grant No. GRK 1355. The work of S. H. G. and T. D. was performed under the auspices of the U.S. Department of Energy by Lawrence Livermore National Laboratory under Contract No. DE-AC52-07NA27344 and was supported by LDRDs 08-ERI-002, 08-LW-004. S. H. G. and C. F. were supported by the Alexander von Humboldt foundation, G. G. partially by the Engineering and Physical Sciences
Research Council (Grant No. EP/G007187/1) and the Science and Technology Facilities Council of the UK. Finally, the authors are greatly indebted to the FLASH team for enabling an outstanding performance.

*thomas.tschentscher@xfel.eu

[1] National Research Council, Frontiers in High Energy Density Physics: The X-Games of Contemporary Science (National Academies Press, Washington, D.C., 2003).

[2] T. Guillot, Science 286, 72 (1999).

[3] N. Nettelmann et al., Astrophys. J. 683, 1217 (2008).

[4] J. D. Lindl et al., Phys. Plasmas 11, 339 (2004).

[5] D. Riley et al., Phys. Rev. Lett. 84, 1704 (2000).

[6] T. Ao et al., Phys. Rev. Lett. 96, 055001 (2006).

[7] S. H. Glenzer and R. Redmer, Rev. Mod. Phys. 81, 1625 (2009).

[8] S. H. Glenzer et al., Phys. Rev. Lett. 90, 175002 (2003).

[9] S. H. Glenzer et al., Phys. Rev. Lett. 98, 065002 (2007).

[10] A. Kritcher et al., Science 322, 69 (2008).

[11] E. Garcia Saiz et al., Nature Phys. 4, 940 (2008).

[12] D. E. Evans and J. Katzenstein, Rep. Prog. Phys. 32, 207 (1969).

[13] A. Höll et al., High Energy Density Phys. 3, 120 (2007).

[14] T. Tschentscher and S. Toleikis, Eur. Phys. J. D 36, 193 (2005).

[15] D. Pines and P. Nozières, The Theory of Quantum Liquids (W.A. Benjamin, New York, 1966).

[16] W. Ackermann et al., Nat. Photon. 1, 336 (2007).

[17] K. Tiedtke et al., New J. Phys. 11, 023029 (2009).

[18] K. Tiedtke et al., J. Appl. Phys. 103, 094511 (2008).

[19] S. Toleikis et al., High Energy Density Phys. 6, 15 (2010).

[20] R. R. Fäustlin et al., JINST 5, P02004 (2010).

[21] P. Beiersdorfer et al., Rev. Sci. Instrum. 70, 276 (1999).

[22] B. Henke, E. Gullikson, and J. Davis, At. Data Nucl. Data Tables 54, 181 (1993).

[23] C. Fortmann et al., High Energy Density Phys. 5, 208 (2009).

[24] J. Chihara, J. Phys. Condens. Matter 12, 231 (2000).

[25] G. Gregori et al., Phys. Rev. E 67, 026412 (2003).

[26] B.E. Warren, X-ray Diffraction (Courier Dover Publications, Mineola, New York, 1990).

[27] G. Gregori, S. Glenzer, and O. Landen, Phys. Rev. E 74, 026402 (2006).

[28] D. Bohm and T. Staver, Phys. Rev. 84, 836 (1951).

[29] A. Ravasio et al., Phys. Rev. Lett. 99, 135006 (2007).

[30] R. Redmer et al., IEEE Trans. Plasma Sci. 33, 77 (2005).

[31] G. Gregori et al., High Energy Density Phys. 3, 99 (2007).

[32] J.D. Huba, NRL Plasma Formulary (Naval Research Laboratory, Washington, D.C., 2009).

[33] M. A. Lieberman and A.J. Lichtenberg, Principles of Plasma Discharges and Materials Processing (John Wiley \& Sons, Inc., New York, N.Y., 1994), 1st ed., p. 71.

[34] W. Hwang, Y.-K. Kim, and M.E. Rudd, J. Chem. Phys. 104, 2956 (1996).

[35] B. Ziaja et al., Eur. Phys. J. D 40, 465 (2006).

[36] B. Ziaja et al., New J. Phys. 11, 103012 (2009).

[37] B. Ziaja et al., Phys. Rev. Lett. 102, 205002 (2009).

[38] R. Mitzner et al., Opt. Express 16, 19909 (2008). 